\title{
Endoscopic ultrasound-guided fine needle biopsy through the interstices of a colonic stent for the diagnosis of metastatic breast cancer using a for- ward-viewing linear echoendoscope
}
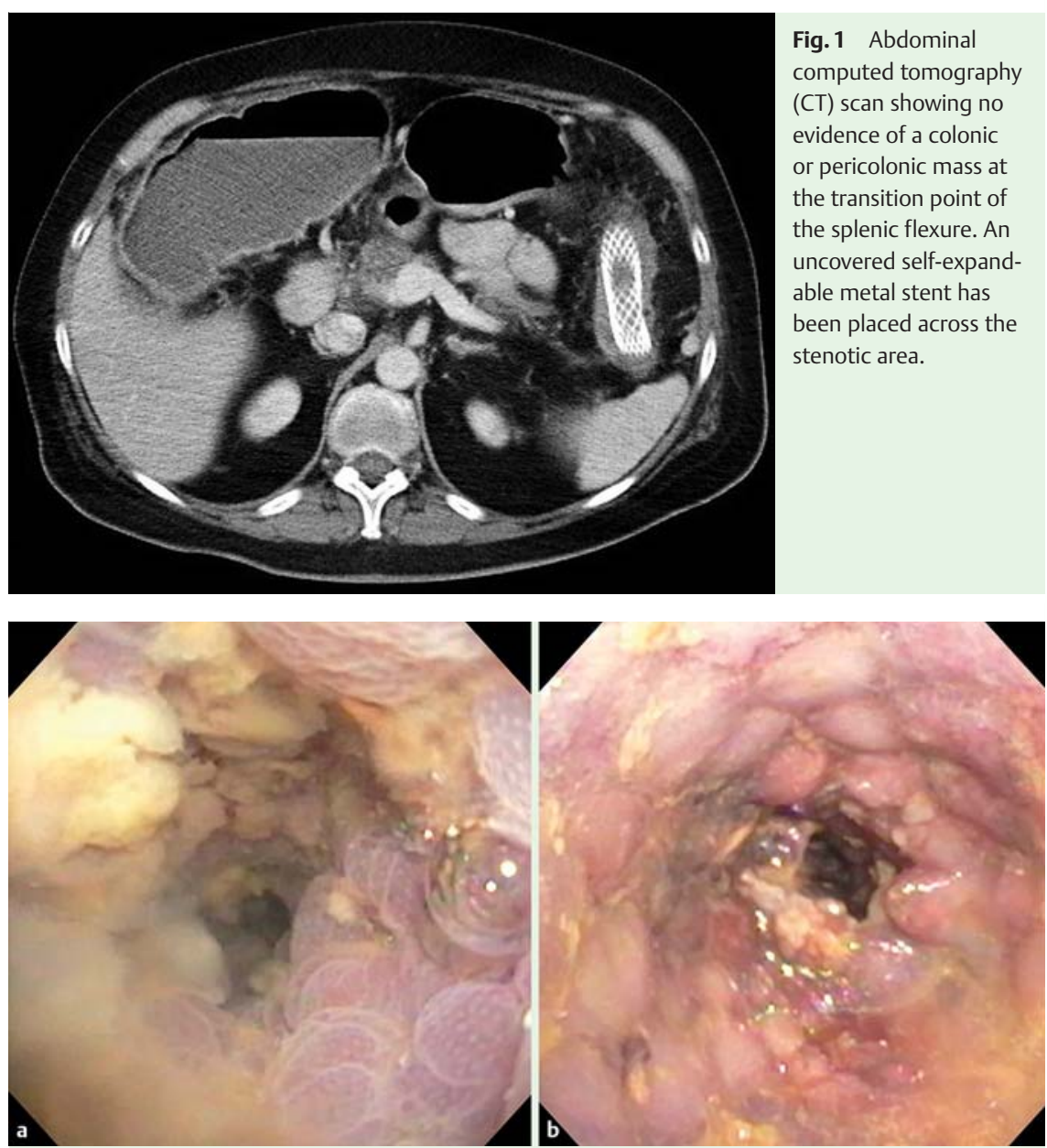

Fig. 2 Endoscopic views showing: a a narrowed lumen in the uncovered self-expandable metal stent; b the narrowing after dilation with a colonic through-the-scope dilator, which allowed the echoendoscope to be passed.

Malignancies of extracolonic origin can be the cause of colorectal obstruction. Acute colorectal obstruction generally requires rapid decompression. Self-expandable metal stent (SEMS) placement is a nonsurgical alternative for palliation of malignant extracolonic obstruction [1]. Knowing the pathology of the underlying cause of the obstruction allows management to be appropriately directed for the stented patient. Pathological confirmation of malignancy by endoscopic biopsy in patients with extracolonic obstruction is difficult and the presence of an existing stent can hamper the ability to obtain diagnostic tissue $[2,3]$.
A 58-year-old woman with a history of inflammatory breast cancer treated nearly 4 years previously with modified radical mastectomy followed by chemotherapy presented with complete colonic obstruction. An abdominal computed tomography (CT) scan showed a transition point at the splenic flexure with cecal dilatation. No evidence was seen of a colonic or pericolonic mass. Flexible sigmoidoscopy was performed, which revealed extrinsic compression causing obstruction. The colonic mucosa appeared normal and no biopsy was taken. An uncovered SEMS (WallFlex; Boston Scientific, Marlborough, Massachusetts, USA) was placed across the ste- notic area ( $\bullet$ Fig. 1 ) and the patient's obstructive symptoms resolved.

The oncology team requested biopsies to define whether recurrent cancer was present as this would allow the patient's enrollment into a chemotherapy trial. A forward-viewing linear echoendoscope (TGF-UC180J; Olympus) [4] was passed into the colon. The SEMS was found in the descending colon and its lumen was seen to be narrowed ( $\bullet$ Fig. 2a). Dilation with a colonic through-the-scope dilator was performed to allow the echoendoscope to pass. There was no endoscopic evidence of visible tumor in the descending colon ( $\bullet$ Fig. 2 b). On endoscopic ultrasound (EUS), diffuse and circumferential low echoic wall thickening was visualized in the descending colon ( $\bullet$ Fig.3a). No surrounding mass or lymphadenopathy was seen. Fine needle biopsy (FNB) was performed without on-site pathologic examination. Six passes were made with a 22-gauge ultrasound-guided core biopsy needle (SharkCore FNB needle; CovidienMedtronic, Minneapolis, Minnesota, USA) through the interstices of the stent ( $\bullet$ Fig. 3b). An additional, Ultraflex precision colonic stent $(25 \times 117 \mathrm{~mm}$; Boston Scientific) was placed through the existing stent.

Standard hematoxylin and eosin (H\&E)stained slides were prepared from formalin-fixed paraffin-embedded biopsy material. Microscopic examination demonstrated cores of colonic mucosa infiltrated by a poorly differentiated carcinoma, consistent with a metastasis from the patient's known breast primary ( $\bullet$ Fig.4).

\section{Endoscopy_UCTN_Code_TTT_1AS_2AZ}

Competing interests: Dr. Baron is a speaker for Medtronic. He is a consultant and speaker for Boston Scientific, Cook Endoscopy, and Olympus, and is a consultant for W.L. Gore. There are no personal conflicts of interest.

\section{Woojin Jeong ${ }^{1,2}$, Dimitri G. Trembath ${ }^{3}$, Todd H. Baron ${ }^{2}$}

${ }^{1}$ Division of Gastroenterology, Department of Internal Medicine, Gangneung Asan Hospital, University of Ulsan College of Medicine, Gangneung, Korea

2 Division of Gastroenterology and Hepatology, University of North Carolina, Chapel Hill, North Carolina, USA

${ }^{3}$ Department of Pathology and Laboratory Medicine, University of North Carolina, Chapel Hill, North Carolina, USA 


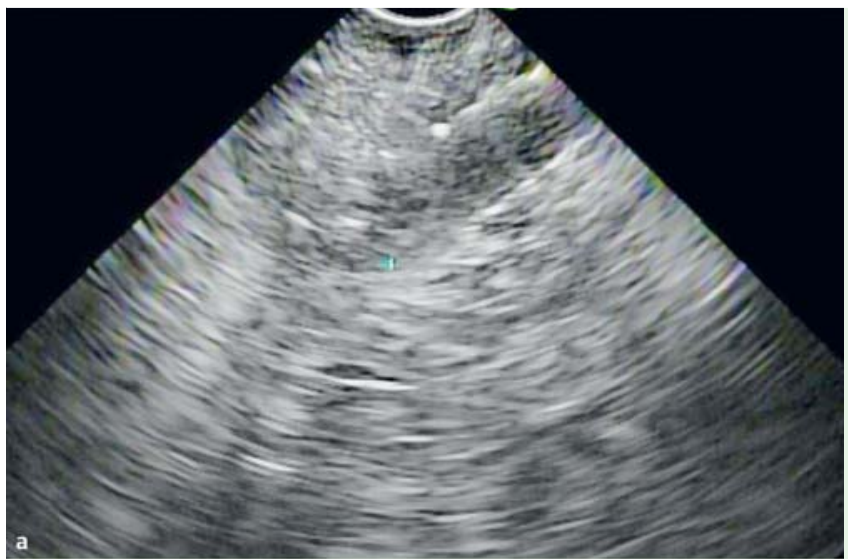

Fig. 3 Endoscopic ultrasound images showing: a a diffuse and circumferential low echoic wall thickening; b a 22-gauge ultrasound core biopsy needle being passed through the interstices of the stent.
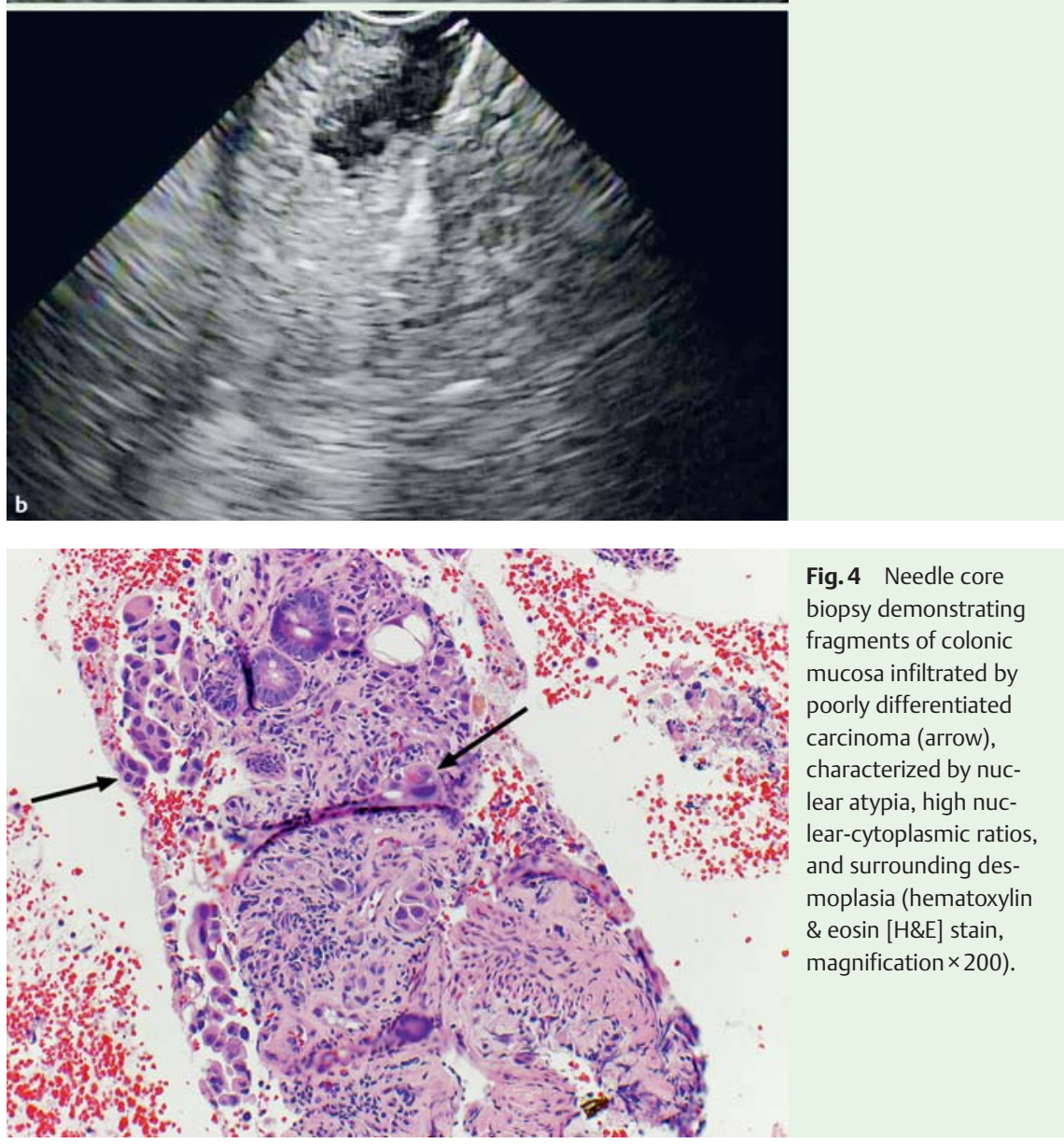

\section{References}

1 van Hooft JE, van Halsema EE, Vanbiervliet G et al. Self-expandable metal stents for obstructing colonic and extra-colonic cancer: ESGE Clinical Guideline. Endoscopy 2014; 46: $990-1002$

2 Prabhu A, Wu M, DiMaio CJ. Successful endoscopic ultrasound-guided fine-needle aspiration through a gastroduodenal stent for the diagnosis of recurrent gallbladder carcinoma. Endoscopy 2015; 47 (Suppl. 01): E109-E110

3 Trindade AJ, Khaitov S, Potack JZ et al. Successful EUS-guided FNA through a colonic stent array for diagnosis of an extraluminal pelvic malignancy causing colonic obstruction. Gastrointest Endosc 2012; 76: 1070 1072

4 Fuccio L, Attili F, Larghi A et al. Forward-viewing linear echoendoscope: a new option in the endoscopic ultrasound armamentarium. J Hepatobiliary Pancreat Sci 2015; 22: 27-34

\section{Bibliography}

DOI http://dx.doi.org/

10.1055/s-0042-110490

Endoscopy 2016; 48: E283-E284

(c) Georg Thieme Verlag KG

Stuttgart · New York

ISSN 0013-726X

\section{Corresponding author}

\section{Todd H. Baron, MD}

Division of Gastroenterology and Hepatology University of North Carolina School of Medicine 101 Manning Drive, Chapel Hill

\section{NC 27514}

United States of America Fax: +1-984-974-0744 todd_baron@med.unc.edu 